\title{
NOMA Aided Interference Management for Full-Duplex Self-Backhauling HetNets
}

\author{
Lei Lei $^{\circledR}$, Eva Lagunas ${ }^{\circledR}$, Symeon Chatzinotas, and Björn Ottersten ${ }^{\circledR}$
}

\begin{abstract}
The presence of mutual-coupled interference in full-duplex self-backhauling heterogeneous networks raises challenges and difficulties in practical scenarios. In this letter, we address this issue by developing a two-tier non-orthogonal multiple access (NOMA) scheme together with efficient power control to enable aggressive frequency reuse and alleviate co-channel interference. For the considered multi-tier and multicell NOMA scenario, we formulate a power minimization problem, and develop an efficient algorithm with guaranteed convergence to enable optimal power control, such that users' data demand is satisfied and backhauling bottleneck is avoided. Numerical results show the fast convergence of the proposed algorithm, and demonstrate that NOMA is in particular favorable for the high-demand cases in power savings.
\end{abstract}

Index Terms-Heterogeneous networks, full-duplex, selfbackhauling, non-orthogonal multiple access.

\section{INTRODUCTION}

$\mathbf{T}$ HE DENSE deployment of small base stations (SBSs) with wireless backhauling on the overlaid macro base stations (MBSs) forms the so-called self-backhauled heterogeneous networks (HetNets). Since the spectrum resource is scarce and expensive, in-band self-backhauling has emerged as an important enabler to balance the costs with scarce frequency resources [1]. In this context, smart duplexing, e.g., fullduplex (FD) operation, and new radio access techniques, e.g., non-orthogonal multiple access (NOMA), are considered to improve the spectral efficiency and ensure certain qualityof-service (QoS). Adopting aggressive frequency reuse, in-band self-backhauling, and FD techniques in HetNets, introduces four types of interference, i.e., cross-tier, inter-cell, intracell interference, and self-interference (see Fig. 1).

Recent progress on FD has enabled suppressing selfinterference to satisfactory levels [2]. Extensive studies have been devoted to resource allocation [3] and performance analysis of outage probability [4] in FD single-cell NOMA. Lei et al. [5] have studied an FD-NOMA-based relaying systems with one MBS and one SBS. In [6], an FD-based massive MIMO system for both downlink and uplink has been investigated. For multi-cell FD NOMA, Elbamby et al. [7] consider utility maximization, and illustrate the performance gains of FD over half-duplex modes. Rate maximization has been widely studied for FD NOMA systems. Due to

Manuscript received March 1, 2018; revised June 5, 2018; accepted June 6, 2018. Date of publication June 15, 2018; date of current version August 10, 2018. This work has been supported by the FNR CORE project ROSETTA (C17/IS/11632107). The associate editor coordinating the review of this letter and approving it for publication was M. Vaezi. (Corresponding author: Lei Lei.)

The authors are with the Interdisciplinary Center for Security Reliability and Trust, University of Luxembourg, L-1855 Luxembourg City, Luxembourg (e-mail: lei.lei@uni.lu; eva.lagunas@uni.lu; symeon.chatzinotas@uni.lu; bjorn.ottersten@uni.lu).

Digital Object Identifier 10.1109/LCOMM.2018.2847672

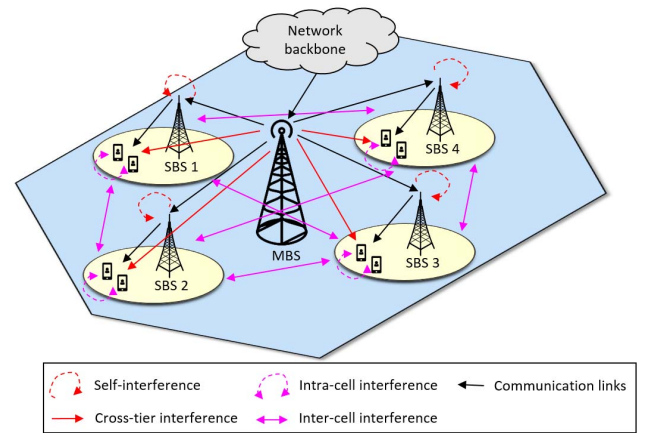

Fig. 1. HetNet scenario with one MBS and four SBSs.

the presence of strong interference in multi-cell networks, power control is also important. Without a proper solution for interference suppression and power control, achieving higher throughput either in MBS or in SBS links would lead to significant increase in power consumption.

In this letter, our contribution lies in addressing two emerging research issues: (1) how much power-saving gains can be expected by using NOMA in the considered FD in-band self-backhauling HetNets, and (2) how to perform effective and efficient interference management such that all the QoS requirements can be satisfied. Firstly, we propose a two-tier NOMA scheme in the system model, in order to facilitate efficient frequency reuse while alleviating the resulting co-channel interference. Secondly, we derive an optimal solution of jointly determining the decoding order in NOMA and the transmit power. The optimization decisions of power and ordering are intertwined and dependent with each other. In solution development, we characterize the dependence and the properties of the optimization variables. Based on the derived proof, we develop an efficient solution to systematically update decoding order and transmit power, which finally leads to an optimal solution with guaranteed convergence. Numerical results verify the competitiveness of using the proposed twotier NOMA in high-demand cases, and the convergence of the proposed power control algorithm.

\section{SySTEM MODEL}

We consider a two-tier self-backhauled HetNet consisting of a single MBS serving $S$ SBSs. All the SBSs are wirelessly connected to the backbone network through the MBS. User equipments' (UEs) traffic is routed through the SBSs nearby. With in-band self-backhauling, all the SBSs and the MBS share the same frequency. All the SBSs operate at the FD mode. Downlink data transmission, i.e., MBS-to-SBSs and SBS-to-UEs, is considered. The basic notations are shown in Table I. The power constraints are stated as follows $P_{m}=\sum_{s=1}^{S} p_{m s}$ and $P_{s}=\sum_{k=1}^{\left|\mathcal{K}_{s}\right|} p_{s k}, \forall s \in\{1, \ldots, S\}$, 
TABLE I

BASIC NOTATIONS

\begin{tabular}{ll}
\hline$P_{m}, P_{s}$ & transmit power at the MBS and the $s$-th SBS \\
$p_{m s}$ & transmit power from the MBS to the $s$-th SBS \\
$p_{s k}$ & transmit power from the $s$-th SBS to UE $k$ \\
$h_{m s}$ & channel coefficient from the MBS to the $s$-th SBS \\
$h_{s k}$ & channel coefficient from the $s$-th SBS to UE $k$ \\
$\hat{h}_{m k}$ & channel coefficient from the MBS to UE $k$ \\
$\hat{h}_{s s^{\prime}}$ & channel coefficient from the $s$-th SBS to the $s^{\prime}$-th SBS \\
$h_{s s}$ & channel coefficient of self-interference at the $s$-th SBS \\
$\sigma_{s}^{2}, \sigma_{k}^{2}$ & noise power at the $s$-th SBS and at UE $k$ \\
\hline
\end{tabular}

where $\mathcal{K}_{s}$ is the set containing all the UEs in the $s$-th SBS. The received interfering signal at each SBS is determined by the inter-cell interference received from other SBSs, the residual self-interference and the interference received from the MBS transmission intended to other SBSs. We denote $0 \leq$ $\beta_{s} \leq 1$ to reflect the $s$-th SBS capability in suppressing its own self-interference [1]-[5]. When $\beta_{s}=1$, no selfinterference cancellation is made, while $\beta_{s}=0$ models perfect self-interference cancellation. The residual self-interference at the $s$-th SBS's receiver is proportional to the transmit power [1]-[5], defined as $\beta_{s} \sum_{k=1}^{\left|\mathcal{K}_{s}\right|} p_{s k}$.

To reduce co-channel interference, we apply NOMA in the MBS-to-SBSs transmission, referred to as "first-tier NOMA". The MBS transmits superposed signals to SBSs, and each SBS has the capability of performing successive interference cancellation (SIC). The SIC decoding order among SBSs is defined as the descending order of $\frac{h_{m 1}}{I_{1}}, \ldots, \frac{h_{m s}}{I_{s}}, \ldots, \frac{h_{m S}}{I_{S}}$ [5], [7], where $I_{s}=h_{s s} \beta_{s} \sum_{k=1}^{\left|\mathcal{K}_{s}\right|} p_{s k}+\sum_{\hat{s} \in \mathcal{S} \backslash\{s\}} P_{\hat{s}} \hat{h}_{\hat{s} s}+\sigma_{s}^{2}$. Suppose two SBSs $s=1,2$ have $\frac{h_{m 1}}{I_{1}} \geq \frac{h_{m 2}}{I_{2}}$. We assume SBS 2's receiver is able to decode its desired signal $x_{2}$. According to the NOMA basis, SBS 1 at its receiver can decode this signal $x_{2}$ only if $\frac{P_{m 2} h_{m 1}}{p_{m 1} h_{m 1}+h_{11} \beta_{1} P_{1}+P_{2} \hat{h}_{21}+\sigma_{1}^{2}} \geq$ $\frac{P_{m 2} h_{m 2}}{p_{m 1} h_{m 2}+h_{22} \beta_{2} P_{2}+P_{1} \hat{h}_{12}+\sigma_{2}^{2}}$, where the former is the SINR of signal $x_{2}$ at SBS 1's receiver, and the latter represents the SINR of signal $x_{2}$ at SBS 2's receiver. One can observe that the inequality holds when $\frac{h_{m 1}}{I_{1}} \geq \frac{h_{m 2}}{I_{2}}$, where $I_{1}=$ $h_{11} \beta_{1} P_{1}+P_{2} \hat{h}_{21}+\sigma_{1}^{2}$ and $I_{2}=h_{22} \beta_{2} P_{2}+P_{1} \hat{h}_{12}+\sigma_{2}^{2}$. As a result, SBS 1 can decode and remove part of the interference, with the rate $\log \left(1+\frac{P_{m 1} h_{m 1}}{h_{11} \beta_{1} P_{1}+P_{2} \hat{h}_{21}+\sigma_{1}^{2}}\right)$. SBS 2 will not be able to perform SIC, achieving the rate $\log (1+$ $\left.\frac{P_{m 2} h_{m 2}}{p_{m 1} h_{m 2}+h_{22} \beta_{2} P_{2}+P_{1} \hat{h}_{12}+\sigma_{2}^{2}}\right)$. Applying the first-tier NOMA, the signal-to-interference-plus-noise ratio (SINR) at the $s$-th SBS can be modeled as $\mathrm{SINR}_{s}^{\mathrm{m}}$. The rate of the MBS towards the $s$-th $\mathrm{SBS}$ is $R_{s}=\log \left(1+\mathrm{SINR}_{s}^{\mathrm{m}}\right)$, where the bandwidth is normalized as 1 , with assuming $\frac{h_{m 1}}{I_{1}} \geq, \ldots, \frac{h_{m s}}{I_{s}}, \ldots, \geq \frac{h_{m S}}{I_{S}}$.

$$
\mathrm{SINR}_{s}^{\mathrm{m}}=\frac{p_{m s} h_{m s}}{h_{s s} \beta_{s} \sum_{k=1}^{\left|\mathcal{K}_{s}\right|} p_{s k}+\sum_{\hat{s}=1}^{s-1} p_{m \hat{s}} h_{m s}+\sum_{\hat{s} \in \mathcal{S} \backslash\{s\}} P_{\hat{s}} \hat{h}_{\hat{s} s}+\sigma_{s}^{2}}, \quad \forall s \in \mathcal{S}
$$

At the transmission of SBS-to-UEs, we introduce "secondtier NOMA" to further mitigate the interference experienced by the UEs. Once the superposed signal from the MBS arrives at each SBS, the SBS decodes and forwards this signal to its associated UEs. At the UEs' side, SIC is applied. Similar to the first-tier NOMA, we sort $\frac{h_{s 1}}{I_{1}^{s}}, \ldots, \frac{h_{s k}}{I_{k}^{s}}, \ldots, \frac{h_{s\left|\mathcal{K}_{s}\right|}}{I_{\left|\mathcal{K}_{s}\right|} \mid}$ by the descending order in the $s$-th SBS, where
$I_{k}^{s}=P_{m} \hat{h}_{m k}+\sum_{\hat{s} \in \mathcal{S} \backslash\{s\}} P_{\hat{s}} h_{\hat{s} k}+\sigma_{k}^{2}, \forall k \in \mathcal{K}_{s}$. Suppose $\frac{h_{s 1}}{I_{1}^{s}} \geq, \ldots, \frac{h_{s k}}{I_{k}^{s}}, \ldots, \geq \frac{h_{s\left|\mathcal{K}_{s}\right|}}{I_{\left|\mathcal{K}_{s}\right|}}$, the SINR of the $k$-th UE in SBS $s$ is shown in $\operatorname{SINR}_{k}^{\mathrm{s}}$. The $k$-th UE receives the cross-tier interference $P_{m} \hat{h}_{m k}$ from the MBS, the intra-cell interference $\sum_{\hat{k}=1}^{k-1} p_{s \hat{k}} h_{s k}$ from the 1 -st to the $k-1$-th UEs, as well as the inter-SBS interference $\sum_{\hat{s} \in \mathcal{S} \backslash\{s\}} P_{\hat{s}} h_{\hat{s} k}$. The $k$-th UE's rate in $\operatorname{SBS} s$ is $R_{k}^{s}=\log \left(1+\operatorname{SINR}_{k}^{s}\right), k \in \mathcal{K}_{s}$.

$$
\operatorname{SINR}_{k}^{\mathrm{s}}=\frac{p_{s k} h_{s k}}{\sum_{\hat{k}=1}^{k-1} p_{s \hat{k}} h_{s k}+P_{m} \hat{h}_{m k}+\sum_{\hat{s} \in \mathcal{S} \backslash\{s\}} P_{\hat{s}} h_{\hat{s} k}+\sigma_{k}^{2}}, \quad \forall k \in \mathcal{K}_{s}
$$

\section{Problem Formulation and Property Analysis}

The addressed two-tier NOMA aided interference management problem contains two components, i.e., determining optimal decoding orders and power allocation. We aim at minimizing the total transmit power over all the BSs in the objective (1a), such that all the UEs' data requests can be satisfied in (1b). In (1c), since the UEs' traffic is routed through the associated SBS, and the SBS has to request the data to the backbone network through the MBS. To avoid the MBS-SBS link becoming a bottleneck, the data rate of the backhauling link is required to be no less than the aggregated rate of the UE access links. To simplify our presentation, we formulate the power control problem under one of the decoding orders, assuming $\frac{h_{m 1}}{I_{1}} \geq, \ldots, \geq \frac{h_{m S}}{I_{S}}$, and $\frac{h_{s 1}}{I_{1}^{s}} \geq$ $, \ldots, \geq \frac{h_{s\left|\mathcal{K}_{s}\right|} \mid}{I_{\left|\mathcal{K}_{s}\right|}^{s}}, \forall s \in \mathcal{S}$ for the moment. The joint optimization of decoding order and power control will be addressed later.

$$
\begin{aligned}
& \text { P1 : } \min _{P_{m}, P_{s}, p_{m s}, p_{s k}} P_{m}+\sum_{s \in \mathcal{S}} P_{s} \\
& \text { s.t. } \log \left(1+\mathrm{SINR}_{k}^{\mathrm{s}}\right) \geq D_{k}, \quad \forall s \in \mathcal{S}, \quad \forall k \in \mathcal{K}_{s} \\
& \sum_{k \in \mathcal{K}_{s}} \log \left(1+\mathrm{SINR}_{k}^{\mathrm{s}}\right) \leq \log \left(1+\mathrm{SINR}_{s}^{\mathrm{m}}\right), \quad \forall s \in \mathcal{S} \\
& P_{m}=\sum_{s=1}^{S} p_{m s}, \quad \text { and } P_{s}=\sum_{k \in \mathcal{K}_{s}} p_{s k}, \quad \forall s \in \mathcal{S} \quad \text { (1c) }
\end{aligned}
$$

The difficulty lies in jointly determining the optimal decoding order and power allocation. These two components mutually influence with each other. The decoding orders in two-tier NOMA vary with transmit power, and the SINR functions in (1b) and (1c) also depend on the decoding orders. Without knowing optimal power, the optimal decoding order is undetermined, and vice versa. Clearly, traversing all the permutations of decoding orders and solving the corresponding problem (P1) under every order, has exponential complexity, and thus inefficient in practice. To solve the joint optimization problem, in Proposition 1 we illustrate how the power $P_{m}, P_{1}, \ldots, P_{S}$ related to each other. We introduce a power vector $\overline{\boldsymbol{p}}_{s}=\left[P_{m}, P_{1}, \ldots, P_{s-1}, P_{s+1}, \ldots, P_{S}\right]$ collecting all the transmit power except the $s$-th SBS's power $P_{s}$.

Proposition 1: $P_{s}$ submits to a closed-form expression of $\overline{\boldsymbol{p}}_{s}$.

Proof: For each UE $k \in \mathcal{K}_{s}$ in the $s$-th SBS, based on $R_{k}^{s}=\log \left(1+\mathrm{SINR}_{k}^{\mathrm{s}}\right)$, we can derive each power variable $p_{s 1}, \ldots, p_{s k}, \ldots, p_{s\left|\mathcal{K}_{s}\right|}$ in the following equations. Suppose 
$\frac{h_{s 1}}{I_{1}^{s}} \geq, \ldots, \geq \frac{h_{s\left|\mathcal{K}_{s}\right|}}{I_{\left|\mathcal{K}_{s}\right|}^{s}}$, the decoding order is consistent with the user index in $\operatorname{SBS} s$.

$$
\begin{aligned}
p_{s 1}= & \left(e^{R_{1}^{s}}-1\right)\left(P_{m} \hat{h}_{m 1}+\sum_{\hat{s} \in \mathcal{S} \backslash\{s\}} P_{\hat{s}} h_{\hat{s} 1}+\sigma_{1}^{2}\right) / h_{s 1}=\left(e^{R_{1}^{s}}-1\right) \frac{I_{1}^{s}}{h_{s 1}} \\
& \ldots \quad\left(\sum_{\hat{k}=1}^{\left|\mathcal{K}_{s}\right|-1} p_{s \hat{k}} h_{s\left|\mathcal{K}_{s}\right|}+I_{\left|\mathcal{K}_{s}\right|}^{s}\right) / h_{s\left|\mathcal{K}_{s}\right|}
\end{aligned}
$$

From the above, all the $p_{s 1}$ appeared in $p_{s 2}, \ldots, p_{s\left|\mathcal{K}_{s}\right|}$ can be replaced by $\left(e^{R_{1}^{s}}-1\right) \frac{I_{1}^{s}}{h_{s 1}}$. Next, each $p_{s 2}$ existed in $p_{s 3}, \ldots, p_{s\left|\mathcal{K}_{s}\right|}$ can be substituted by $\left(e^{R_{2}^{s}}-1\right)\left(\left(e^{R_{1}^{s}}-\right.\right.$ 1) $\left.\frac{I_{1}^{s}}{h_{s 1}} h_{s 2}+I_{2}^{s}\right) / h_{s 2}$, and so on, until substituting $p_{s,\left|\mathcal{K}_{s}\right|-1}$ in $p_{s\left|\mathcal{K}_{s}\right|}$. Once this substituting process is complete, we are able to explicitly express $P_{s}=p_{s 1}+, \ldots,+p_{s\left|\mathcal{K}_{s}\right|}$ in (2) by the function of $P_{m}, P_{1}, \ldots, P_{s-1}, P_{s+1}, \ldots, P_{S}$, hence the conclusion

$$
\begin{aligned}
P_{s}= & -\frac{P_{m} \hat{h}_{m\left|\mathcal{K}_{s}\right|}+\sum_{\hat{s} \in \mathcal{S} \backslash\{s\}} P_{\hat{s}} h_{\hat{s}\left|\mathcal{K}_{s}\right|}+\sigma_{\left|\mathcal{K}_{s}\right|}^{2}}{h_{s\left|\mathcal{K}_{s}\right|}}+\sum_{k=1}^{\left|\mathcal{K}_{s}\right|}\left[e^{\sum_{\hat{k}=k}^{\left|\mathcal{K}_{s}\right|} R_{\hat{k}}^{s}}\right. \\
& \left.\times\left(\frac{P_{m} \hat{h}_{m k}+\sum_{\hat{s} \in \mathcal{S} \backslash\{s\}} P_{\hat{s}} h_{\hat{s} k}+\sigma_{k}^{2}}{h_{s k}}\right)\right] \\
= & \quad \sum_{k=1} \frac{P_{m} \hat{h}_{m, k-1}+\sum_{\hat{s} \in \mathcal{S} \backslash\{s\}} P_{\hat{s}} h_{\hat{s}, k-1}+\sigma_{k-1}^{2}}{h_{s, k-1}}\left(\frac{I_{k}^{s}}{h_{s k}}-\frac{I_{k-1}^{s}}{h_{s, k-1}}\right) e^{\sum_{\hat{k}=k}^{\left|\mathcal{K}_{s}\right|} R_{\hat{\kappa}}^{s}}-\frac{I_{\left|\mathcal{K}_{s}\right|}^{s}}{h_{s\left|\mathcal{K}_{s}\right|}} \\
= & f\left(P_{m}, P_{1}, \ldots, P_{s-1}, P_{s+1}, \ldots, P_{S}\right)
\end{aligned}
$$

In Proposition 1, we reveal the dependence between $P_{s}$ and $\left[P_{m}, P_{1}, \ldots, P_{s-1}, P_{s+1}, \ldots, P_{S}\right]$. Next, we characterize the function $P_{s}=f\left(\overline{\boldsymbol{p}}_{s}\right)$ by introducing the concept of standard interference function (SIF). By definition, if a function $f(\boldsymbol{x})$ : $\mathbb{R}_{+}^{n} \rightarrow \mathbb{R}_{+}^{n}$, for all the input $\boldsymbol{x}$, satisfies the following three properties: positivity, $f(\boldsymbol{x})>0$; monotonicity, if $\boldsymbol{x}^{\prime} \geq \boldsymbol{x}$ then $f\left(\boldsymbol{x}^{\prime}\right) \geq f(\boldsymbol{x})$; and scalability, $\alpha f(\boldsymbol{x})>f(\alpha \boldsymbol{x})$ for all $\alpha>1$, then $f(\boldsymbol{x})$ is SIF. Starting from any initial point and performing fixed-point iteration based algorithm, the convergence of the proposed method is guaranteed [8].

Proposition 2: $f\left(\overline{\boldsymbol{p}}_{s}\right), \forall s \in \mathcal{S}$, is SIF.

Proof: From the proof of Proposition 1, the positivity can be observed. For monotonicity, we increase all the elements in $\overline{\boldsymbol{p}}_{s}$ by a positive value $\epsilon>0$, i.e., $\overline{\boldsymbol{p}}_{s}^{\prime}=\overline{\boldsymbol{p}}_{s}+\epsilon$, and substitute all the entities $P_{m}, P_{1}, \ldots, P_{s-1}, P_{s+1}, \ldots, P_{S}$ in (2) by $P_{m}+\epsilon$, $P_{1}+\epsilon, \ldots, P_{s-1}+\epsilon, P_{s+1}+\epsilon, \ldots, P_{S}+\epsilon$. As a result, all the values of $p_{s 1}, p_{s 2}, \ldots, p_{s\left|\mathcal{K}_{s}\right|}$ are strictly increased. Since $P_{s}=\sum_{k \in \mathcal{K}_{s}} p_{s k}$ then $f\left(\overline{\boldsymbol{p}}_{s}^{\prime}\right)>f\left(\overline{\boldsymbol{p}}_{s}\right)$. In terms of scalability, let $\alpha>1$ and derive $\alpha f\left(\overline{\boldsymbol{p}}_{s}\right)$ and $f\left(\alpha \overline{\boldsymbol{p}}_{s}\right)$ as follows,

$$
\begin{aligned}
& \alpha f\left(\overline{\boldsymbol{p}}_{s}\right) \\
& =\left(e^{R_{1}^{s}}-1\right)\left(\alpha P_{m} \hat{h}_{m 1}+\sum_{\hat{s} \in \mathcal{S} \backslash\{s\}} \alpha P_{\hat{s}} h_{\hat{s} 1}+\alpha \sigma_{1}^{2}\right) / h_{s 1}+, \ldots, \\
& \quad+\frac{e^{R_{\left|\mathcal{K}_{s}\right|}^{s}-1}}{h_{s\left|\mathcal{K}_{s}\right|}}\left(\alpha \sum_{\hat{k}=1}^{\left|\mathcal{K}_{s}\right|-1} p_{s \hat{k}} h_{s\left|\mathcal{K}_{s}\right|}+\alpha P_{m} \hat{h}_{m\left|\mathcal{K}_{s}\right|}\right. \\
& \left.\quad+\alpha \sum_{\hat{s} \in \mathcal{S} \backslash\{s\}} P_{\hat{s}} h_{\hat{s}\left|\mathcal{K}_{s}\right|}+\alpha \sigma_{\left|\mathcal{K}_{s}\right|}^{2}\right)
\end{aligned}
$$

$$
\begin{aligned}
& f\left(\alpha \overline{\boldsymbol{p}}_{s}\right) \\
& =\left(e^{R_{1}^{s}}-1\right)\left(\alpha P_{m} \hat{h}_{m 1}+\sum_{\hat{s} \in \mathcal{S} \backslash\{s\}} \alpha P_{\hat{s}} h_{\hat{s} 1}+\sigma_{1}^{2}\right) / h_{s 1}+, \ldots, \\
& \quad+\frac{e^{R_{\left|\mathcal{K}_{s}\right|}}-1}{h_{s\left|\mathcal{K}_{s}\right|}}\left(\sum_{\hat{k}=1}^{\left|\mathcal{K}_{s}\right|-1} p_{s \hat{k}} h_{s\left|\mathcal{K}_{s}\right|}+\alpha P_{m} \hat{h}_{m\left|\mathcal{K}_{s}\right|}\right. \\
& \left.\quad+\alpha \sum_{\hat{s} \in \mathcal{S} \backslash\{s\}} P_{\hat{s}} h_{\hat{s}\left|\mathcal{K}_{s}\right|}+\sigma_{\left|\mathcal{K}_{s}\right|}^{2}\right)
\end{aligned}
$$

It can be observed that $\alpha f\left(\overline{\boldsymbol{p}}_{s}\right)>f\left(\alpha \overline{\boldsymbol{p}}_{s}\right)$. Hence the conclusion follows.

Analogously to the proof of Proposition 1 and 2, we reach the following similar results for $P_{m}$ in Corollary 3.

Corollary 3: $P_{m}$ is a function of $\overline{\boldsymbol{p}}_{m}$, and $P_{m}=f\left(\overline{\boldsymbol{p}}_{m}\right)$ is SIF.

Proof: Assuming $\frac{h_{m 1}}{I_{1}} \geq, \ldots, \frac{h_{m s}}{I_{s}}, \ldots, \geq \frac{h_{m S}}{I_{S}}, P_{m}$ can be expressed by a closed-form function $f\left(\overline{\boldsymbol{p}}_{m}\right)$ in (3), where $\overline{\boldsymbol{p}}_{m}=\left[P_{1}, \ldots, P_{S}\right]$ and $P_{m}=f\left(\overline{\boldsymbol{p}}_{m}\right)$.

$$
P_{m}=\sum_{s=1}^{S}\left(\frac{I_{s}}{h_{m s}}-\frac{I_{s-1}}{h_{m, s-1}}\right) e^{\sum_{\hat{s}=s}^{S} R_{\hat{s}}}-\frac{I_{S}}{h_{m S}}=f\left(\overline{\boldsymbol{p}}_{m}\right)
$$

The property of SIF follows analogously.

Remark 1: The three properties of SIF in $f\left(\overline{\boldsymbol{p}}_{m}\right)$ and $f\left(\overline{\boldsymbol{p}}_{s}\right)$ are independent with the SIC decoding order. Thus, $f\left(\overline{\boldsymbol{p}}_{m}\right)$ and $f\left(\overline{\boldsymbol{p}}_{s}\right)$ are SIF under any of possible decoding orders.

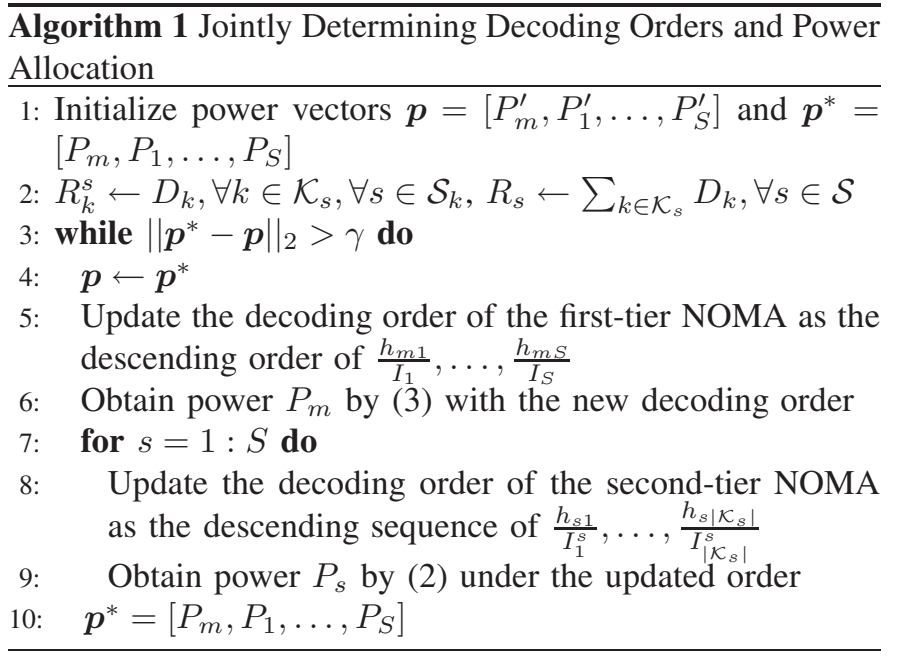

By our analysis, thus far we are able to develop a fixedpoint iteration based algorithm, see Algorithm 1, to jointly optimize the two-tier NOMA decoding orders and the power allocation. That is, we adjust $P_{m}, P_{1}, \ldots, P_{S}$ one by one. When a power variable is updating, all the others remain fixed. Even though the power adjustment in a cell has mutualinfluence effect to the other cells, having proven the property of SIF, this procedure will eventually converge to a fixed power point which leads to the optimum [8]. Algorithm 1 terminates when the Euclidean distance between the power vectors of two successive iterations, given by the 2-norm $\left\|\boldsymbol{p}^{*}-\boldsymbol{p}\right\|_{2}$, is smaller than a tolerate value $\gamma=10^{-5}$. 
TABLE II

SIMULATION PARAMETERS

\begin{tabular}{ll}
\hline Parameter & Value \\
Cell radius & $300 \mathrm{~m}$ \\
Carrier frequency & $2 \mathrm{GHz}$ \\
Bandwidth & $1 \mathrm{MHz}$ \\
Number of UEs & 2,5 \\
Number of MBS and SBSs & 1 and 4 \\
Coefficient $\beta_{s}$ & 0.5 \\
Channel model & ITU urban macro/micro-cell model \\
Noise power & $-173 \mathrm{dBm} / \mathrm{Hz}$ \\
\hline
\end{tabular}

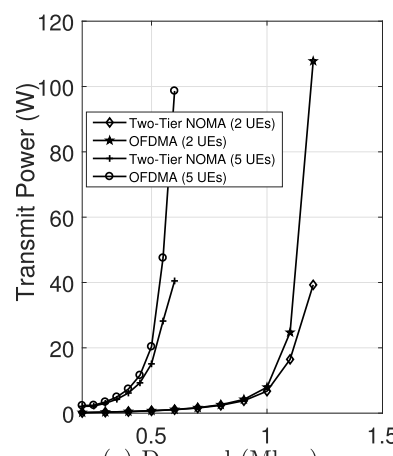

(a) Demand (Mbps)

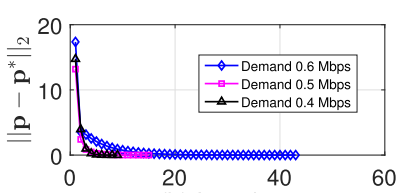

(b) Iterations

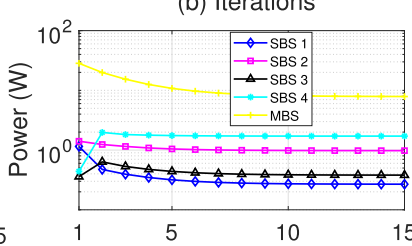

(c) Iterations
Fig. 2. (a), total power with respect of demand and number of UEs; (b), evolution of convergence in Alg. 1; (c), evolution of power in all the cells.

\section{Numerical Results}

We present numerical results to illustrate the performance of the two-tier NOMA scheme and the algorithm, in terms of power savings and convergence. The simulation parameters are summarized in Table II. An optimal power allocation scheme for orthogonal frequency division multiple access (OFDMA) networks, presented in [9], is implemented for comparison. To enable a fair comparison between FD-OFDMA and FD-NOMA, we require that all the cells in [9] are active, and all the SBSs are operating at the FD mode. The revised algorithm provides an optimal power solution for full-duplex based OFDMA HetNets. All the numerical results are averaged over 1000 realizations.

In Fig. 2a and Table III, compared with [9], we show the power-saving gains due to applying NOMA and the proposed Algorithm 1. The two-tier NOMA demonstrated its superiority, evidenced by consistently less power consumption than OFDMA. We observe that the performance gains of NOMA over OFDMA is relatively marginal for the lowdemand cases, less than $10 \%$, while for the high-demand instances, NOMA starts to demonstrate its significant gains of power savings which can achieve 1.5-2 times power reduction than OFDMA. This also means that NOMA yields better capability in supporting UEs' increasing demand. In practice, the total power consumption also grows successively with increase of number of UEs and BSs, and the level of residual self-interference [3], [5]. In contrast, power increases exponentially with the UEs' demand, and thus it is considered as a dominant factor in the simulation.

Next, in Fig. 2b, we reveal the evolution of convergence in Algorithm 1. We use the cases of 5 UEs per SBS for illustration. For the three different demands, in average the convergence is reached after around 8, 14, 43 iterations, respectively. When the demand increases, more iterations are required for convergence. In addition, from Table III, the
TABLE III

Summary of the Performance IN Fig. 2A

\begin{tabular}{cccccc}
\hline Demand (2 UEs): & 0.6 & 0.8 & 1.0 & 1.1 & 1.2 \\
\hline Gaps & $4.2 \%$ & $7.3 \%$ & $17.7 \%$ & $50.1 \%$ & $174.3 \%$ \\
Ava. Iter. & 6.2 & 8.3 & 11.9 & 21.3 & 41.8 \\
\hline Demand (5 UEs): & 0.4 & 0.45 & 0.5 & 0.55 & 0.6 \\
Gaps & $19.6 \%$ & $25.0 \%$ & $34.9 \%$ & $68.5 \%$ & $143.6 \%$ \\
Ava. Iter. & 8.2 & 9.6 & 14.1 & 25.4 & 43.1 \\
\hline
\end{tabular}

number of UEs also influences the required iterations to converge, but the effect appears moderate. In general, Algorithm 1 is capable of converging fast. In Fig. 2c, we illustrate the evolution of transmit power of SBSs and the MBS in the algorithm's execution. The cases of demand $=0.5 \mathrm{Mbps}$ are used. All the cells' transmit power can be successively reduced over iterations until reaching the convergence, although the effect of interference suppression may not necessarily be monotonic. By applying Algorithm 1, the mutual-coupled ICI can be eventually suppressed or maintained at an optimal level without causing performance degradation to the other parts. In the meanwhile, the system consumes the minimum power to satisfy all the constraints in $\mathbf{P 1}$.

\section{CONCLUSION}

We have developed an efficient iterative algorithm with guaranteed optimality and convergence for the NOMA aided FD in-band self-backhauling HetNets. The proposed two-tier NOMA scheme and the iterative algorithm are able to significantly reduce the required power consumption than OFDMA in particular for the cases of high demand. A promising extension of the current work is to investigate the potential gains of power control under the imperfections of NOMA, e.g., imperfect channel estimation and SIC, as well as developing efficient distributed algorithms for interference management. Another extension is to incorporate the SIC delay NOMA in FD operation and investigate the time-domain resource scheduling.

\section{REFERENCES}

[1] A. Sabharwal et al., "In-band full-duplex wireless: Challenges and opportunities," IEEE J. Sel. Areas Commun., vol. 32, no. 9, pp. 1637-1652, Sep. 2014.

[2] M. S. Sim et al., "Nonlinear self-interference cancellation for full-duplex radios: From link-level and system-level performance perspectives," IEEE Commun. Mag., vol. 55, no. 9, pp. 158-167, Jun. 2017.

[3] Y. Sun et al., "Optimal joint power and subcarrier allocation for full-duplex multicarrier non-orthogonal multiple access systems," IEEE Trans. Commun., vol. 65, no. 3, pp. 1077-1091, Mar. 2017.

[4] Z. Mobini et al., "Full-duplex multi-antenna relay assisted cooperative non-orthogonal multiple access," in Proc. IEEE GLOBECOM, Dec. 2017, pp. 1-7.

[5] L. Lei et al., "Energy optimization for full-duplex self-backhauled HetNet with non-orthogonal multiple access," in Proc. IEEE SPAWC, Jul. 2017, pp. 1-5.

[6] X. Xia et al., "Beam-domain full-duplex massive MIMO: Realizing co-time co-frequency uplink and downlink transmission in the cellular system," IEEE Trans. Veh. Technol., vol. 66, no. 10, pp. 8845-8862, Oct. 2017.

[7] M. S. Elbamby et al., "Resource optimization and power allocation in in-band full duplex-enabled non-orthogonal multiple access networks," IEEE J. Sel. Areas Commun., vol. 35, no. 12, pp. 2860-2873, Dec. 2017.

[8] R. D. Yates, "A framework for uplink power control in cellular radio systems," IEEE J. Select. Areas Commun., vol. 13, no. 7, pp. 1341-1347, Sep. 1995.

[9] L. Lei et al., "Optimal cell clustering and activation for energy saving in load-coupled wireless networks," IEEE Trans. Wireless Commun., vol. 14, no. 11, pp. 6150-6163, Nov. 2015. 